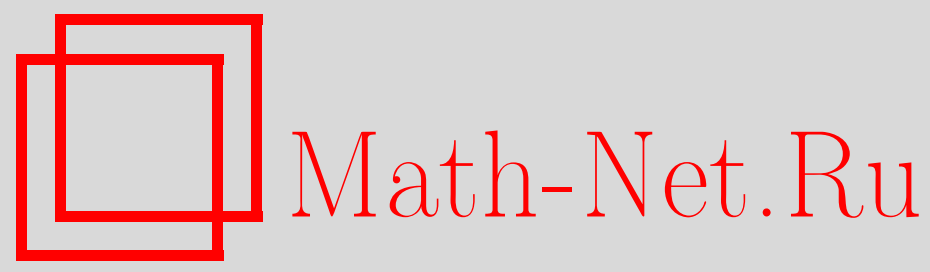

В. К. Белошапка, Универсальная модель вещественного подмногообразия, Матем. заметки, 2004, том 75, выпуск $4,507-522$

DOI: https://doi.org/10.4213/mzm49

Использование Общероссийского математического портала Math-Net.Ru подразумевает, что вы прочитали и согласны с пользовательским соглашением http://www.mathnet.ru/rus/agreement

Параметры загрузки:

IP : 54.205 .225 .156

26 апреля 2023 г., 04:31:26 


\title{
УНИВЕРСАЛЬНАЯ МОДЕЛЬ ВЕЩЕСТВЕННОГО ПОДМНОГООБРАЗИЯ
}

\section{В.К. Белошапка}

\begin{abstract}
В предыдущих работах автора была развита технология вычисления автоморфизмов, построения инвариантов и классификаций вещественных подмногообразий комплексного пространства. Основным ее этапом является построение "хорошей" модельной поверхности. Хорошая модельная поверхность - это аналог соприкасающегося параболоида классической дифференциальной геометрии. Модельные поверхности, предложенные ранее, обладали полньм списком нужных свойств лишш при соблюдении некоторой оценки сверху на коразмерность многообразия. Если эта оценка нарушалась, то поверхности теряли свойство универсальности (способность правильно коснуться любого ростка), что ограничивало сферу их применимости. В данной работе это ограничение снимается: для произвольного типа $(n, K)(n$ - размерность комплексной касательной, $K$ - вещественная коразмерность) строится хорошая модельная поверхность. В частности, решена задача построения невырожденного ростка вещественно-аналитического подмногообразия комплексного пространства произвольного фиксированного типа $(n, K)$ с самой богатой в данном классе группой голоморфных автоморфизмов.
\end{abstract}

Библиография: 28 названий.

\section{1. Введение}

Теория функций нескольких комплексных переменных начинается с $\mathbb{C}^{2}$. Один из способов продемонстрировать отличие $\mathbb{C}^{2}$ от $\mathbb{C}^{1}$ заключается в том, чтобы показать, что в $\mathbb{C}^{2}$ почти любая малая деформация шара приводит к области, голоморфно шару не эквивалентной, тогда как слегка помятый диск в $\mathbb{C}^{1}$ останется эквивалентным непомятому (теорема Римана). Это различие можно сформулировать как утверждение о различии локальных свойств границ областей: два любых вещественно-аналитических подмногообразия $\mathbb{C}^{1}$ коразмерности один (т.е. две кривые) локально эквивалентны, а в $\mathbb{C}^{2}$ такие же подмногообразия (т.е. трехмерные гиперповерхности) - почти всегда нет (это в том или ином виде было известно А. Пуанкаре, см. также [1]). Говорят, что с ростом размерности растет аналитическая жесткость. Жесткость ростка данного подмногообразия в точке (точнее, ее отсутствие) можно измерять богатством локальной группы голоморфных автоморфизмов (точнее, ее размерностью). Для кривой в $\mathbb{C}^{1}$ эта

Работа выполнена при финансовой поддержке Российского фонда фундаментальных исследований, гранты № 0015-96-008 и № 02-01-01-291. 
величина всегда равна бесконечности, тогда как для ростков трехмерных вешественно-аналитических подмногообразий в $\mathbb{C}^{2}$ ситуация следующая. Для вещественной гиперплоскости мы получаем бесконечность. Если росток не эквивалентен гиперплоскости, то эта размерность не превосходит восьми. Восемь мы имеем для стандартной трехмерной сферы. Если росток не эквивалентен сфере, то эта размерность не превосходит трех. Тройку дают гиперповерхности, однородные в общей точке. Картан [2] написал список таких гиперповерхностей. Гиперповерхность общего положения автоморфизмов вообще не имеет, даже локальных.

Таким образом, гиперплоскость и гиперсфера оказываются решениями двух экстремальных задач: гиперплоскость реализует абсолютньй максимум размерности, а сфеpa - максимум в классе конечномерных групп. В силу этого легко предположить, что эти две гиперповерхности будут играть исключительную роль в данном круге вопросов. Так оно и есть: имеется разработанная технология (метод модельной поверхности), ориентированная на описание отображений, автоморфизмов, инвариантов и классификацию ростков вешественных подмногообразий, ключом к применению которой является построение “хорошей” модельной поверхности [3], [4]. Для $\mathbb{C}^{2}$ такая поверхностьэто наша сфера.

Если перейти от $\mathbb{C}^{2}$ к гиперповерхности в пространстве произвольной размерности, то полньм аналогом сферы являются некоторые специальные гиперповерхности второго порядка (их несколько, в положительно определенном случае это гиперсфера) [5], [28], [3]. Как было показано в работе автора [6] 1991-го года, прямым обобщением сферы для многообразий коразмерности более высокой, чем единица, являются "невырожденные квадрики" - специальные поверхности, определяемые уравнениями второго порядка. При условии положительной определенности эти поверхности фигурировали ранее в теории однородных областей как остовы областей Зигеля второго рода. Квадрикам и изучению вещественных многообразий с опорой на эту модель посвящена обширная литература (см. список в [4]). Однако “квадрики” вьполняют роль модельных поверхностей лишь для не слишком высоких коразмерностей (точное условие таково: коразмерность не превосходит квадрата размерности комплексной касательной). Для поверхностей более высокой коразмерности такие же полные аналоги сферы были найдены в предыдущих работах автора и Шананиной [7]-[12], [4]. Граница на коразмерность была отодвинута, но бесконечности не достигла. Хорошие модельные поверхности были построены для весьма широкого спектра ситуаций, однако для “очень высоких" значений коразмерности они, все же, были не пригодны. При “очень высоких" значениях коразмерности утрачивалось свойство универсальности, которое состоит в том, что модельной поверхностью можно “правильно" коснуться любого невырожденного ростка. В данной работе приводится полное решение задачи о построении хорошей модельной поверхности.

Процесс порождения модельных поверхностей при фиксированной размерности комплексной касательной $n$ все более высоких коразмерностей напоминает процесс заполнения электронами разрешенных энергетических уровней в атоме. При этом вся серия напоминает периодическую систему элементов Менделеева. Первьй период образуют квадрики. Они являются хорошими модельными поверхностями для коразмерностей от 1 до $n^{2}$ ( $n$ - размерность комплексной касательной). Последняя в периоде поверхность (она в каждом периоде единственна) соответствует инертному газу. Дальше идет скачек степени (новый энергетический уровень) и т.д. 
Построенные поверхности являются "хорошими” модельными поверхностями (см. теорему 14) по отношению к классу вполне невырожденных ростков (см. определение 1). Если же ставить задачу построения системы модельных поверхностей, охватьвающей и случаи вырождения, то такая задача естественно порождает древовидную классификацию ростков и моделей, для которой построенная в данной работе система является стволом.

\section{2. Процесс построения модельной поверхности произвольного типа}

Фиксируем произвольные значения $n \geqslant 1$ и $K \geqslant 1$. Пара $(n, K)$ - это тип вещественного порождающего многообразия, т.е. $n$ - это размерность комплексной касательной, так назьваемая CR-размерность, а $K$ - вещественная коразмерность. Пусть $z \in \mathbb{C}^{n}$, присвоим этой переменной вес равный единище: $[z]=1$. В процессе построения мы будем постепенно увеличивать $d$ - степень уравнений, определяющих нашу поверхность, до тех пор, пока она не окажется достаточно высокой для выбранного значения $K$. Процесс, формально, можно начать с первого шага $(d=1)$, но этот шаг приводит к пустому множеству уравнений и переменньх, поэтому мы его пропускаем и начинаем сразу со второго шага $(d=2)$.

В дальнейшем через $\left\langle x_{1}^{a_{1}} \ldots x_{N}^{a_{N}}\right\rangle$ будем обозначать произвольньй вектор-многочлен (размерность будет ясна из контекста) от переменных $x_{1}, \ldots, x_{N}$, однородньй по $x_{j}$ степени $a_{j}$; если же нам потребуется как-либо выделить один из таких многочленов, мы будем писать $P\left(x_{1}^{a_{1}} \ldots x_{N}^{a_{N}}\right)$. Через $O(m)$ будем обозначать выражения, содержащие члены веса не менњшего, чем $m$.

2.1. Второй шаг. Рассмотрим пространство $\mathscr{F}_{2}$ вещественных многочленов от $(z, \bar{z})$ веса 2 . Зададим произвольное $\tilde{k}_{2} \geqslant 1$, введем в рассмотрениепеременную $w_{2}=u_{2}+i v_{2} \in$ $\mathbb{C}^{\tilde{k}_{2}}$ и рассмотрим поверхность $\widetilde{Q}\left(n, \tilde{k}_{2}\right)$, заданную в $\mathbb{C}^{n} \oplus \mathbb{C}^{\tilde{k}_{2}}$ соотношением

$$
v_{2}=\widetilde{\Phi}_{2}(z, \bar{z})
$$

где каждая координата $\Phi_{2}$ принадлежит $\mathscr{F}_{2}$. При этом $\widetilde{\Phi}_{2}=\langle z \bar{z}\rangle+2 \operatorname{Re}\left\langle z^{2}\right\rangle$, т.е. имеет место разложение $\mathscr{F}_{2}=\mathscr{N}_{2} \oplus \mathscr{H}_{2}$, где $\mathscr{N}_{2}-$ пространство эрмитовых форм вида $\langle z \bar{z}\rangle$, а $\mathscr{H}_{2}$ - пространство гармонических форм вида $2 \operatorname{Re}\left\langle z^{2}\right\rangle$. Члены вида $2 \operatorname{Re}\left\langle z^{2}\right\rangle$ легко убрать заменой $w \rightarrow w+2 i\left\langle z^{2}\right\rangle$ и уравнение $\widetilde{Q}_{2}$ принимает вид

$$
v_{2}=\Phi_{2}(z, \bar{z})
$$

где каждая координата $\Phi_{2}$ принадлежит пространству эрмитовых форм $\mathfrak{N}_{2}$. Мы говорим, что $\Phi_{2}$ - это результат нормализации $\widetilde{\Phi}_{2}$.

Наложим на $\Phi_{2}$ условие невырожденности. Невырожденность формы $\Phi_{2}$ означает линейную независимость координатных форм и отсутствие ядра, т.е. если для всех $z$ $\Phi_{2}(\zeta, \bar{z})=0$, то $\zeta=0$. Обозначим через $k_{2}$ размерность $\mathcal{N}_{2}$. Из условия линейной независимости следует, что $\tilde{k}_{2} \leqslant k_{2}$. Если $K \leqslant k_{2}$, то положим $Q(n, K)=\widetilde{Q}(n, K)$, объявим $Q(n, K)$ модельной поверхностью типа $(n, K)$ и завершим процесс построения. Если же $K>k_{2}$, то положим $\tilde{k}_{2}=k_{2}$, присвоим переменным $w_{2}, u_{2}$ вес 2 и перейдем к выполнению следующего третьего шага.

Прежде, чем дать описание третьего шага, приведем список объектов и величин, полученных в результате выполнения второго шага:

пространство гармонических форм веса $2-\mathscr{H}_{2}$; 
пространство нормализованных форм веса $2-\mathscr{N}_{2} ;$

$k_{2}-$ размерность $\mathscr{N}_{2}$

пространство $\mathbb{C}^{k_{2}}$ переменных $w_{2}=u_{2}+i v_{2}$ веса 2 ;

условие невырожденности формы $\Phi_{2} \in \mathscr{N}_{2}$.

Отметим, что если $K \leqslant k_{2}$, то построенная модельная поверхность является хорошо известной невырожденной квадрикой [6], [4].

2.2. Третий шаг. Рассмотрим пространство $\mathscr{F}_{3}$ вещественных многочленов от $(z, \bar{z}$, $u_{2}$ ) веса 3 . Зададим произвольное $\tilde{k}_{3} \geqslant 1$, введем в рассмотрение переменную $w_{3}=$ $u_{3}+i v_{3} \in \mathbb{C}^{\tilde{k}_{3}}$, присвоим переменным $w_{3}, u_{3}$ вес 3 и рассмотрим поверхность $\widetilde{Q}\left(n, k_{2}, \tilde{k}_{3}\right)$, заданную в $\mathbb{C}^{n} \oplus \mathbb{C}^{k_{2}} \oplus \mathbb{C}^{\tilde{k}_{3}}$ соотношением

$$
\begin{aligned}
& v_{2}=\Phi_{2}(z, \bar{z}), \\
& v_{3}=\widetilde{\Phi}_{3}\left(z, \bar{z}, u_{2}\right),
\end{aligned}
$$

где форма $\Phi_{2}$ принадлежит $\mathscr{N}_{2}$ и невырождена в смысле определения, данного выше, a форма $\widetilde{\Phi}_{3}$ принадлежит $\mathscr{F}_{3}$. Условие на $\Phi_{2}$ означает, что координаты $\Phi_{2}-$ это базис пространства $\mathscr{N}_{2}$. Любые два таких базиса эквивалентны с точностью до вещественной линейной замены переменной $w_{2}$. В этой ситуации условие тривиальности ядра становится следствием линейной независимости координатных форм.

Многочлен из $\mathscr{F}_{3}$ имеет следующий общий вид:

$$
\widetilde{\Phi}_{3}=2 \operatorname{Re}\left(\left\langle z^{3}\right\rangle+\left\langle z^{2} \bar{z}\right\rangle+\left\langle z u_{2}\right\rangle\right) .
$$

Пространство $\mathscr{H}_{3}$ порождается гармоническими мономами веса 3 от $z$ и $w_{2}=u_{2}+$ $i \Phi_{2}(z, \bar{z})$, т.е. мономами вида $\operatorname{Re}\left\langle z^{3}\right\rangle$ и $\operatorname{Re}\left\langle z\left(u_{2}+i \Phi_{2}\right)\right\rangle$. При построении прямого дополнения к $\mathscr{H}_{3}$ - подпространства $\mathscr{N}_{3}$ - возникает неоднозначность. Можно при нормализации уничтожить члены вида $\operatorname{Re}\left\langle z u_{2}\right\rangle$, a $\operatorname{Re}\left\langle z^{2} \bar{z}\right\rangle$ оставить, а можно наоборот. Мы всегда будем руководствоваться следующим правилом: нормализация идет в направлении уменьшения степени старшей переменной. В данной ситуации это $u_{2}$ и, пользуясь свободой в выборе гармонического слагаемого, мы уничтожаем члены вида $\operatorname{Re}\left\langle z u_{2}\right\rangle, \mathrm{a}$ $\operatorname{Re}\left\langle z^{2} \bar{z}\right\rangle$ оставляем. Соответственно $\mathscr{N}_{3}$ - это пространство вешественных кубических форм вида $\operatorname{Re}\left\langle z^{2} \bar{z}\right\rangle$, и имеет место разложение $\mathscr{F}_{3}=\mathscr{N}_{3} \oplus \mathscr{H}_{3}$.

После полиномиальной замены поверхность $\widetilde{Q}\left(n, k_{2}, \tilde{k}_{3}\right)$ можно представить в виде

$$
\begin{aligned}
& v_{2}=\Phi_{2}(z, \bar{z}), \\
& v_{3}=\Phi_{3}\left(z, \bar{z}, u_{2}\right),
\end{aligned}
$$

где $\Phi_{3}\left(z, \bar{z}, u_{2}\right) \in \mathcal{N}_{3}$. Наложим на $\Phi_{3}$ условие невырожденности. Невырожденность формы $\Phi_{3}$ означает просто линейную независимость координатньх форм. Обозначим через $k_{3}$ размерность $\mathscr{N}_{3}$. Из условия линейной независимости следует, что $\tilde{k}_{3} \leqslant k_{3}$. Если $K \leqslant k_{2}+k_{3}$, то положим $Q(n, K)=\widetilde{Q}\left(n, k_{2}, K-k_{2}\right)$, объявим $Q(n, K)$ модельной поверхностью типа $(n, K)$ и завершим процесс построения. Если же $K>k_{2}+k_{3}$, то перейдем к выполнению следующего шага.

На этом шаге, как и на предыдущем, был сформирован следующий список объектов, величин и условий:

пространство гармонических форм веса $3-\mathscr{H}_{3}$; 
пространство нормализованных форм веса $3-\mathscr{N}_{3}$;

$k_{3}$ - размерность $\mathscr{N}_{3}$

пространство $\mathbb{C}^{k_{3}}$ переменных $w_{3}=u_{3}+i v_{3}$ веса 3 ;

условие невырожденности формы $\Phi_{3} \in \mathscr{N}_{3}$.

Если $K \leqslant k_{2}+k_{3}$, то построенная поверхность $Q(n, K)$ является невырожденной кубикой, построенной в [9].

Отметим следующее. Условия невырожденности второго и третьего шагов не совпадают. Условие второго шага, кроме условия линейной независимости, содержит требование тривиальности ядра. С третьего шага условие тривиальности ядра эрмитовой формы поглощается условием линейной независимости этих форм, и все дальнейшие условия - это условие линейной независимости координатных форм после нормализации. Поэтому мы не включаем условие невырожденности в список конструируемых объектов.

Теперь мы готовы дать описание произвольного шага нашего процесса.

2.3. $(m+1)$-й шаг. На предыдушем, $m$-м шаге был сфформирован следующий список объектов:

пространство гармонических форм веса $m-\mathscr{H}_{m}$;

пространство нормализованных форм веса $m-\mathscr{N}_{m}$;

$k_{m}$ - размерность $\mathscr{N}_{m}$

пространство $\mathbb{C}^{k_{m}}$ переменньх $w_{m}=u_{m}+i v_{m}$ веса $m$.

Рассмотрим пространство $\mathscr{F} m+1$ вещественных многочленов от $\left(z, \bar{z}, u_{2}, \ldots, u_{m}\right)$ веса $m+1$. Зададим произвольное $\tilde{k}_{m+1} \geqslant 1$, введем в рассмотрение переменную $w_{m+1}=$ $u_{m+1}+i v_{m+1} \in \mathbb{C}^{\tilde{k}_{m+1}}$, присвоим переменньм $w_{m+1}, u_{m+1}$ вес $m+1$ и рассмотрим поверхность $\widetilde{Q}\left(n, k_{2}, \ldots, k_{m}, \tilde{k}_{m+1}\right)$, заданную в $\mathbb{C}^{n} \oplus \mathbb{C}^{k_{2}} \oplus \cdots \oplus \mathbb{C}^{k_{n}} \oplus \mathbb{C}^{\tilde{k}_{m+1}}$ соотношением

$$
\begin{aligned}
v_{2} & =\Phi_{2}(z, \bar{z}), \\
\ldots & \ldots \ldots \ldots, \\
v_{m} & =\Phi_{m}\left(z, \bar{z}, u_{2}, \ldots, u_{m-1}\right), \\
v_{m+1} & =\widetilde{\Phi}_{m+1}\left(z, \bar{z}, u_{2}, \ldots, u_{m}\right),
\end{aligned}
$$

где $\Phi_{j} \in \mathscr{N}_{j}$ при $j=1, \ldots, m$, а $\widetilde{\Phi}_{m+1} \in \mathscr{F}_{m+1}$. Условия невырожденности, введенные на предыдущих шагах, означают, что координатные формы $\Phi_{j}$ - это базис пространства $\mathcal{N}_{j}$.

Список гармонических мономов $L_{m+1}$ состоит из мономов вида $\operatorname{Re}\left\langle z^{p} w_{2}^{p_{2}} \ldots w_{m}^{p_{m}}\right\rangle$, где $1 p+2 p_{2}+\cdots+m p_{m}=m+1$ и $w_{j}=u_{j}+i \Phi_{j}$. Подпространство $\mathscr{H}_{m+1}-$ это линейная оболочка $L_{m+1}$. Пространство $\mathscr{N}_{m+1}-$ прямое дополнение $\mathscr{H}_{m+1}$ до $\mathscr{F}_{m+1}-$ выбираем из условия минимизации степени старшей переменной $k_{m+1}=\operatorname{dim} \mathscr{N}_{m+1}$.

После полиномиальной замены уравнения поверхности $\widetilde{Q}\left(n, k_{2}, \ldots, \tilde{k}_{m+1}\right)$ принимают вид

$$
\begin{aligned}
& v_{2}=\Phi_{2}(z, \bar{z}) \\
& \ldots \ldots \ldots \ldots \ldots, \\
& v_{m}=\Phi_{m}\left(z, \bar{z}, u_{2}, \ldots, u_{m-1}\right),
\end{aligned}
$$




$$
v_{m+1}=\Phi_{m+1}\left(z, \bar{z}, u_{2}, \ldots, u_{m}\right)
$$

где $\Phi_{m+1} \in \mathscr{N}_{m+1}$. Наложим на $\Phi_{m+1}$ условие невырожденности. Невырожденность $\Phi_{m+1}$ означает линейную независимость координатных форм. Из условия линейной независимости следует, что $\tilde{k}_{m+1} \leqslant k_{m+1}$. Если $K \leqslant k_{2}+\cdots+k_{m+1}$, то положив

$$
Q(n, K)=\widetilde{Q}\left(n, k_{2}, \ldots, k_{m}, K-\left(k_{2}+\cdots+k_{m}\right)\right),
$$

объявим $Q(n, K)$ модельной поверхностью типа $(n, K)$ и остановим процесс построения. Если же нет, то перейдем к выполнению следующего шага.

Поскольку все размерности $k_{j}$ положительны, процесс закончится за конечное число шагов. Можно показать, что $k_{j}$ растут с ростом $j$ экспоненциальным образом. Номер последнего шага обозначим через $d(n, K)$. Это старшая степень полиномиальных уравнений, определяющих поверхность $Q(n, K)$. Эта целочисленная функция медленно (логарифмически) растет с ростом $K$ и убывает с ростом $n$ [10]. Условие окончания процесса - это выполнение неравенства

$$
k_{2}+\cdots+k_{d-1}<K \leqslant k_{2}+\cdots+k_{d} .
$$

Введем еще один параметр

$$
k=K-\left(k_{2}+\cdots+k_{d-1}\right),
$$

он изменяется в пределах от 1 до $k_{d}$. Тогда $Q(n, K)$ - это поверхность в $\mathbb{C}^{n} \oplus \mathbb{C}^{k_{2}} \oplus \cdots$ $\oplus \mathbb{C}^{k_{d-1}} \oplus \mathbb{C}^{k}$, заданная соотношениями

$$
\begin{aligned}
v_{2} & =\Phi_{2}(z, \bar{z}), \\
\ldots \ldots & \ldots \ldots \ldots \ldots, \\
v_{d-1} & =\Phi_{d-1}\left(z, \bar{z}, u_{2}, \ldots, u_{d-2}\right), \\
v_{d} & =\Phi_{d}\left(z, \bar{z}, u_{2}, \ldots, u_{d-1}\right) .
\end{aligned}
$$

Здесь координаты $\Phi_{j}$ при $j=2, \ldots, d-1$ - это базис $\mathscr{N}_{j}$, а координаты $\Phi_{d}-$ линейно независимые элементы $\mathscr{N}_{d}$.

Выполняя вьшеописанньй процесс до седьмого шага можно убедиться в том, что

$$
\begin{aligned}
\mathscr{N}_{3}= & \left\{2 \operatorname{Re}\left\langle z^{2}, \bar{z}\right\rangle\right\}, \\
\mathscr{N}_{4}= & \left\{2 \operatorname{Re}\left(\left\langle z^{3}, \bar{z}\right\rangle+\left\langle z^{2}, \bar{z}^{2}\right\rangle\right)\right\}, \\
\mathscr{N}_{5}= & \left\{2 \operatorname{Re}\left(\left\langle z^{4}, \bar{z}\right\rangle+\left\langle z^{3}, \bar{z}^{2}\right\rangle+\left\langle z^{2}, \bar{z}, u_{2}\right\rangle\right)\right\}, \\
\mathscr{N}_{6}= & \left\{2 \operatorname{Re}\left(\left\langle z^{5}, \bar{z}\right\rangle+\left\langle z^{4}, \bar{z}^{2}\right\rangle+\left\langle z^{3}, \bar{z}^{3}\right\rangle+\left\langle z^{3}, \bar{z}, u_{2}\right\rangle+\left\langle z^{2}, \bar{z}^{2}, u_{2}\right\rangle\right)\right\}, \\
\mathscr{N}_{7}= & \left\{2 \operatorname { R e } \left(\left\langle z^{6}, \bar{z}\right\rangle+\left\langle z^{5}, \bar{z}^{2}\right\rangle+\left\langle z^{4}, \bar{z}^{3}\right\rangle+\left\langle z^{4}, \bar{z}, u_{2}\right\rangle+\left\langle z^{3}, \bar{z}^{2}, u_{2}\right\rangle\right.\right. \\
& \left.\left.+\left\langle z^{2}, \bar{z}, u_{2}{ }^{2}\right\rangle+\left\langle z^{3}, \bar{z}, u_{3}\right\rangle+\left\langle z^{2}, \bar{z}^{2}, u_{3}\right\rangle\right)\right\} .
\end{aligned}
$$

Это определяет вид модельных поверхностей соответствующих степеней.

Модельная поверхность фиксированного типа не обязана быть единственной, даже если не различать биголоморфно эквивалентные экземпляры. Такая ситуация имела место уже для квадрик $(d=2)$. Однако если $k=k_{d}$, то единственность (с точностью до эквивалентности) имеется.

Если исходная поверхность была задана уравнениями вида $v=\phi(z, \bar{z})$ (отсутствует переменная $u$, такие поверхности иногда называют “жесткими”), то описанная процедура приводит к модельной поверхности такого же вида. Эти поверхности были описаны и изучены ранее [10], [11]. 


\section{3. Поверхность $Q(n, K)$ является хорошей модельной поверхностью произвольного вполне невырожденного ростка типа $(n, K)$}

С каждым ростком $M_{\xi}$ можно связать алгебру Ли ростков векторных полей, голоморфных в окрестности $\xi$ и касательных к поверхности в точках поверхности. Эту алгебру мы обозначаем через aut $M_{\xi}$, соответствующую ей локальную групу голоморфных автоморфизмов через Aut $M_{\xi}$; подгрупу автоморфизмов, сохраняющих $\xi$ на месте и ее алгебру Ли $-\operatorname{Aut}_{\xi} M_{\xi}$ и aut $_{\xi} M_{\xi}$ соответственно.

Рассмотрим в пространстве $\mathbb{C}^{n} \oplus \mathbb{C}^{k_{2}} \oplus \cdots \oplus \mathbb{C}^{k_{d-1}} \oplus \mathbb{C}^{k}$ росток гладкой поверхности типа $(n, K)$, заданньй в начале координат локальными уравнениями

$$
\begin{aligned}
v_{2} & =\Phi_{2}(z, \bar{z})+O(3), \\
\ldots \ldots \ldots \ldots \ldots \ldots \ldots, & \left.\ldots \ldots, u_{d-2}\right)+O(d), \\
v_{d-1} & =\Phi_{d-1}\left(z, \bar{z}, u_{2}, \ldots, u_{d-1}\right)+O(d+1), \\
v_{d} & =\Phi_{d}\left(z, \bar{z}, u_{2}, \ldots, u, \ldots\right.
\end{aligned}
$$

где $\Phi_{j} \in \mathscr{N}_{j}$. Поверхность, заданную соотношениями

$$
\begin{aligned}
v_{2} & =\Phi_{2}(z, \bar{z}) \\
\ldots \ldots \ldots \ldots \ldots & \left.\ldots \ldots, u_{d-2}\right), \\
v_{d-1} & =\Phi_{d-1}\left(z, \bar{z}, u_{2}, \ldots,\right. \\
v_{d} & =\Phi_{d}\left(z, \bar{z}, u_{2}, \ldots, u_{d-1}\right),
\end{aligned}
$$

обозначим $Q(n, K)$ или просто $Q$ и будем называть модельной поверхностью.

ОПРЕДЕЛЕНИЕ 1. Говорим, что росток задан в стандартном виде или в стандартных координатах, если его уравнения имеют вид $(1)$, причем координаты $\Phi_{j}$ при $j=2, \ldots, d-1$ - это базис $\mathscr{N}_{j}$, а координаты $\Phi_{d}$ - линейно независимые элементы $\mathscr{N}_{d}$. Говорим, что модельная поверхность $Q$, заданная младшими членами (1), является модельной поверхностью ростка $M_{\xi}$. Говорим, что росток $M_{\xi}$ вполне невьрожден, если в некоторых координатах он имеет стандартный вид; при этом говорим, что модельная поверхность $Q$ невырождена.

Описанньй вьше алгоритм построения модельной поверхности является одновременно и алгоритмом приведения порождающего ростка произвольного типа $(n, K)$ к стандартному виду. Действительно, на каждом шаге мы подавали на вход произвольные многочлены соответствующего веса. При приведении конкретного ростка это будут весовые компоненты тейлоровского разложения уравнений ростка. Единственное препятствие в работе алгоритма - это появление на каком-то этапе нулевой нормализованной компоненты. Если этого не произошло (это и есть наше условие вполне невырожденности), то алгоритм закончит свою работу, записав уравнения ростка в виде (1). Переход к стандартным координатам осуществляется обратимой полиномиальной заменой. Поэтому мы можем сформулировать утверждение, которое, по сушеству, является тавтологией.

ПРЕДЛОЖЕНИЕ 2. Любой вполне невырожденный росток обратимой полиномиальной заменой можнно привести к стандартному виду. 
ПРЕДЛОЖЕНИЕ 3. В группе Aut $Q$ голоморфных автоморфизмов поверхности $Q$ имеется подгруппа Aut_ $Q$ полиномиально-треугольных автоморфизмов степени $d-1$, действующая на $Q$ транзитивно и без неподвижных точек. Поверхность $Q$ является, таким образом, изоморфной группе Ли Aut_ $Q$ и, в частности, является однородной.

ДокАЗАтЕЛЬСтво. Рассмотрим преобразование

$$
z \rightarrow p+z, \quad w \rightarrow q+w
$$

где $(p, q)$ - произвольная точка поверхности $Q$. Уравнение поверхности $Q$ при этом изменится. Многочлен старшего веса $\Phi_{d}\left(z, \bar{z}, u_{2}, \ldots, u_{d-1}\right)$ после этой подстановки переходит в выражение

$$
\Phi_{d}\left(z, \bar{z}, u_{2}, \ldots, u_{d-1}\right)+H \Phi_{d}+N \Phi_{d},
$$

где $H \Phi_{d}$ - гармонические члены, а $N \Phi_{d}$ - нормализованные члены, причем весовое разложение $H \Phi_{d}+N \Phi_{d}$ содержит лиш компоненты весов, строго меньших $d$. Все гармонические члены убираются полиномиальной заменой вида $w_{d} \rightarrow w_{d}+P\left(z, w_{2}, \ldots, w_{d-1}\right)$, где $P$ - многочлен без свободного члена, а нормализованные - линейной заменой вида $w_{d} \rightarrow w_{d}+L\left(w_{2}, \ldots, w_{d-1}\right)$. В результате последнее уравнение возвращается к исходному виду. Аналогичное преобразование предпоследнего уравнения не затрагивает переменной $w_{d}$ и последнего уравнения. Действуя последовательно таким образом, возвращаем все уравнения к исходному виду, т.е. композиция отображений является автоморфизмом, переводящим $(0,0)$ в $(p, q)$. При этом данное преобразование единственно и имеет вид

$$
z \rightarrow z+p, \quad w_{j} \rightarrow q_{j}+w_{j}+P_{j}\left(z, w_{2}, \ldots, w_{j-1}\right), \quad j=2, \ldots, d,
$$

где степени полиномов не превосходят $d-1$. Такие преобразования называются треугольными. Доказательство закончено.

Отметим, что для квадратичных модельных поверхностей, т.е. при $d=2$ эта группа была известна как группа Гейзенберга и являлась подгруппой аффинной группы.

Распространим нашу градуировку и на векторные поля, полагая

$$
\left[\frac{\partial}{\partial z}\right]=-1, \quad\left[\frac{\partial}{\partial \bar{z}}\right]=-1, \quad\left[\frac{\partial}{\partial w_{j}}\right]=-j, \quad\left[\frac{\partial}{\partial \bar{w}_{j}}\right]=-j .
$$

Пусть $g_{-}-$это алгебра Ли групшы Aut_ $Q$. Простое вычисление дает следующее

ПРЕДЛОЖЕНИЕ 4. (а) Алгебра $g_{-}$- это градуированная алгебра Ли вида $g_{-}=$ $g_{-d}+\cdots+g_{-1}$.

(b) Алгебра $g_{-}$порохдается компонентой $g_{-1}$; точнее, каждая компонента $g_{-m}, m=2, \ldots, d$, есть линейная комбинация скобок полей из $g_{-1}$ степени $m$.

(c) При отождествлении $Q \cong$ Aut_ $Q$ пространство $T_{0}^{c} Q$ отождествляется c $g_{-1}$, а вся алгебра $g_{-}$с алгеброй Леви-Танаки модельной поверхности $Q$.

(d) Выполнено

$$
g_{-1} \cong \mathbb{C}^{n}, \quad g_{-j} \cong \mathbb{R}^{k_{j}} \quad \text { npu } j=2, \ldots, d-1, \quad g_{-d} \cong \mathbb{R}^{k} .
$$

В частности, поверхность $Q$ является поверхностью сильно регулярной по Танаке [5].

Определение вполне невырожденности можно связать с другими характеристиками вещественного ростка следующим образом. 
ПРЕДЛОЖЕНИЕ 5. (а) Вполне невырожденный росток является ростком конечного типа по Кону [13].

(b) В терминологии Блума-Грэхма [14] вполне невырожденный росток является ростком типа

$$
(2, \ldots, 2,3, \ldots, 3, \ldots, d, \ldots, d)
$$

где двоек $-k_{2}$ штук, троек - $k_{3}$ штук и т.д., стариее значение $d$ представлено в количестве $k$ штук.

(c) Вполне невырожденный росток является d-стабильным [15].

(d) Вполне невырожденный росток минимален в смысле Туманова [16].

(е) В терминах работ Баоуенди, Эбенфельта, Хуанга и Ротиильд [17], [18] вполне невырожденный росток является 1-невырожсденным.

ДокАЗАТЕльСтво. (a) и (b) следуют из результатов п. 8 работы $[14],(\mathrm{c})$ и (d) - из предложения $4,($ e) - непосредственно из определения $q$-невырожденности.

В работе Блума и Грэхма координатное определение типа связано с геометрическим описанием в терминах касательных полей и их коммутаторов. Если $L_{1}$ - это пространство ростков векторных полей, которые в каждой точке принадлежат комплексной части касательной, то можно рассмотреть градуированную алгебру Ли (алгебра Леви-Танаки)

$$
L=\bigcup_{j=1}^{\infty} L_{j}, \quad L_{j+1}=\left[L_{j}, L_{1}\right]+L_{j}
$$

Конечность типа в нашей ситуации означает, что $L_{d}=L$ и значения полей из $L_{d}$ в центре ростка совпадают со всем касательньм пространством. Таким образом, степень $d$ модельной поверхности совпадает с длиной градуированной алгебры. Вполненевырожденность в этих терминах означает, что каждьй раз при переходе к коммутаторам более высокой степени происходит максимально возможньй рост размерности пространства значений полей в центре ростка.

Пусть $M_{\xi}$ и $\widetilde{M}_{\tilde{\xi}}-$ два ростка одного типа $(n, K)$, уравнения которых в стандартных координатах соответственно имеют вид

$$
\begin{aligned}
& \operatorname{Im} w_{2}=\Phi_{2}(z, \bar{z})+O(3), \quad \operatorname{Im} w_{2}=\widetilde{\Phi}_{2}(z, \bar{z})+O(3), \\
& \text {.............. } \\
& \operatorname{Im} w_{d}=\Phi_{d}(z, \bar{z})+O(d+1), \quad \operatorname{Im} w_{d}=\widetilde{\Phi}_{d}(z, \bar{z})+O(d+1) .
\end{aligned}
$$

ПРЕДЛОЖЕНИЕ 6. Если $\phi=\left(f, g_{2}, \ldots, g_{d}\right), \phi(0)=0,-$ локальная голоморфная әквивалентность мехду $M_{\xi}$ и $\widetilde{M}_{\tilde{\xi}}$, то

$$
f=C z+O(2), \quad g_{j}=\rho_{j} w_{j}+O(j+1), \quad j=2, \ldots, d,
$$

әде $C \in G L(n, \mathbb{C}), \rho_{j} \in G L\left(k_{j}, \mathbb{R}\right), j=2, \ldots, d-1, \rho_{d} \in G L(k, \mathbb{R})$, причем для всех $j$ имеют место тождества

$$
\widetilde{\Phi}_{j}\left(C z, \overline{C z}, \rho_{2} u_{2}, \ldots, \rho_{j-1} u_{j-2}\right)=\rho_{j} \Phi_{j}\left(z, \bar{z}, u_{2}, \ldots, u_{j-1}\right) .
$$


ДокАЗАТЕЛЬСтво. Вводя в рассмотрение младшие компоненты координат отображения и записьвая то, что $\phi$ переводит $M_{\xi}$ в $\widetilde{M}_{\tilde{\xi}}$, получаем

$$
\begin{aligned}
& \operatorname{Im}\left(\langle z\rangle+\rho_{2} w_{2}+O(3)\right)=\Phi_{2}(C z+O(2), \overline{C z+O(2)})+O(3), \\
& \operatorname{Im}\left(\langle z\rangle+\left\langle z^{2}\right\rangle+\left\langle w_{2}\right\rangle+\rho_{3} w_{3}+O(4)\right)=\Phi_{3}(C z+O(2), \overline{C z+O(2)})+O(4), \\
& \operatorname{Im}\left(\langle z\rangle+\cdots+\rho_{4} w_{4}\right)+O(5)=\Phi_{4}(C z+O(2), \overline{C z+O(2)})+O(5), \\
& \operatorname{Im}\left(\langle z\rangle+\cdots+\rho_{d} w_{d}+O(d+1)\right) \\
& =\Phi_{d}\left(C z+O(2), \overline{C z+O(2)}, \operatorname{Re}\left(g_{2}\right), \ldots, \operatorname{Re}\left(g_{d-1}\right)\right)+O(d+1) .
\end{aligned}
$$

Выделяя первую весовую компоненту во всех координатах, получаем, что во всех координатах $\langle z\rangle=0$. Выделяя вторую весовую компоненту во всех координатах, получаем, что для всех $g_{j}, j=2, \ldots, d$, выполнено

$$
\left\langle z^{2}\right\rangle=0, \quad \operatorname{Im} \rho_{2}=0, \quad \rho_{2} \Phi_{2}(z, \bar{z})=\widetilde{\Phi}_{2}(C z, \overline{C z})
$$

и для всех координат от $g_{3}$ до $g_{d}$ члены $\left\langle w_{2}\right\rangle=0$. Сравнивая далее весовые компоненты от третьей до $d$-ой, получаем утверждение теоремы.

В качестве следствия получаем такое утверждение.

СлЕДСТВИЕ 7. Если два ростка әквивалентны, то әквивалентны и их модельные поверхности. Две модельных поверхности голоморфно әквивалентны тогда и только тогда, когда они әквивалентны линейно. Точнее это означает, что найдутся невырожденные линейные преобразования $C, \rho_{2}, \ldots, \rho_{d}$ такие, что

$$
\rho_{j} \Phi_{j}(z, \bar{z})=\widetilde{\Phi}_{j}(C z, \overline{C z}), \quad j=2, \ldots, d .
$$

Таким образом, на множестве модельных поверхностей типа $(n, K)$ действует группа

$$
G L(n, \mathbb{C}) \oplus G L\left(k_{2}, \mathbb{R}\right) \oplus \cdots \oplus G L\left(k_{d-1}, \mathbb{R}\right) \oplus G L(k, \mathbb{R}),
$$

которая естественным образом редуцируется к действию

$$
G L(n, \mathbb{C}) \oplus G L(k, \mathbb{R}),
$$

и в силу следствия 7 все инварианты этого действия являются CR-инвариантами ростка. Для квадратичных моделей этот сюжет был развит в работах [19]-[22].

Невырожденные модельные поверхности, как было отмечено вьше, 1-невырождены, минимальны и вещественно алгебраичны, поэтому к ним применима теорема Зайцева [23], из которой получаем

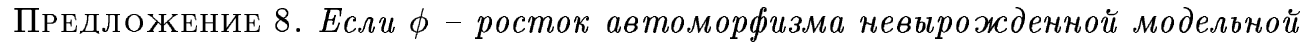
поверхности $Q(n, K)$ в точке $\xi \in Q(n, K)$, то ф аналитически зависит от своей $2(K+1)$-струи в точке $\xi$ ияляется, таким образом, конечномерной группой Ли в естественной топологии.

Это утверждение можно дополнить следующим очевидным замечанием.

ЗАмЕчАниЕ 9. Если поверхность $Q(n, K)$ вырождена (т.е. правые части уравнений линейно зависимы), то ее группа автомор физмов является бесконечномерной. 
ПРЕДЛОЖЕНИЕ 10. Алгебра Ли aut $Q(n, K)$ инфинитезимальных автоморфизмов поверхности $Q(n, K)$ - это подалгебра полиномиальных векторных полей, степени которых равномерно ограничены константой, зависящей лишь от $n$ и $K$.

ДокАЗАТЕльство. Конечномерность групш влечет за собой конечномерность алгебры. Векторные поля, образующие aut $Q$, имеют вид

$$
\operatorname{Re}\left(f \frac{\partial}{\partial z}+g_{2} \frac{\partial}{\partial w_{2}}+\cdots+g_{d} \frac{\partial}{\partial w_{d}}\right)
$$

где коэффициенты $f, g_{2}, \ldots, g_{d}$ голоморфны в окрестности нуля. Рассмотрим разложение этой алгебры Ли в сумму градуированных компонент. Имеет место следующее непосредственно проверяемое свойство: если некоторое поле содержится в aut $Q$, то каждая его градуированная компонента - тоже. Таким образом, предположение о существовании полей с мономами сколь угодно высоких степеней противоречит конечномерности алгебры. Это завершает доказательство.

Для алгебры инфинитезимальных автоморфизмов модельной поверхности имеет место разложение aut $Q=g_{-}+g_{0}+g_{+}$, где $g_{-}=g_{-d}+\cdots+g_{-1}$ обсуждалась в предложении 4 , размерность этой подалгебры равна размерности $Q$, т.е. $2 n+K$. Подалгебре $g_{0}$ соответствует подгруппа линейных автоморфизмов $Q$ - LAut $Q$, сохраняющих начало координат на месте (см. предложение 6 и следствие 7). Размерность этой подгрупшы не превосходит $2 n^{2}$ (по матрице $C$ преобразование определяется однозначно). При $k=k_{d}$ этот максимум достигается, в общем случае размерность гораздо меньше, но не меньше единищы, так как эта подгруппа в любом случае содержит преобразования вида

$$
z \in t z, \quad w_{j} \in t^{j} w_{j}, \quad j=2, \ldots, d, \quad t \in \mathbb{R} .
$$

Подалгебре aut $+Q=g_{1}+\cdots+g_{D}$ соответствует подгруппа Aut $+Q$ нелинейных преобразований $Q$, сохраняющих начало координат на месте. Пусть $D=D(n, K)-$ это максимальньй вес полей, входящих в алгебру. Если $d=2$ (это соответствует условию $K \leqslant n^{2}$ ), то $D \leqslant 2$. Вопрос о существовании модельньх поверхностей с нетривиальной подгруппой Aut $+Q$ открыт уже при $d=3$. В этом случае имеется оценка $D \leqslant 6$ (см. [11]). Несмотря на имеющуюся в этом вопросе неясность, можно с помощю стандартных аргументов [24], [6], [9], [10] получить следующее

ПРЕДЛОЖЕНИЕ 11. Автоморфизмы невырожденной модельной поверхности $Q(n, K)$ - это бирациональные преобразования $\mathbb{C}^{n+K}$, степени которых равномерно ограничены константой, зависящей лишь от $n$ и $\mathrm{K}$.

Столь же дословно на рассматриваемый общий случай переносится доказательство следующего утверждения.

ПРЕДЛОЖЕНИЕ 12. Пусть $M_{\xi}$ - росток вполне невырожденного вещественного подмногообразия класса $C^{\infty}$, a $Q$-его касательная модельная поверхность. Тогда

$$
\operatorname{dim} \text { Aut } M_{\xi} \leqslant \operatorname{dim} \text { Aut } Q, \quad \operatorname{dim}_{\operatorname{Aut}_{\xi}} M_{\xi} \leqslant \operatorname{dim} \operatorname{Aut}_{0} Q .
$$

ДокАЗАТЕЛЬСтво буквально повторяет все доказательства аналогичных оценок (см. [16], [6], [9], [10]) и представляет собой некую версию теоремы о неявных функциях в формальных рядах.

Также доказьвается следующий усиленньй вариант этого утверждения. 
ПРЕДЛОЖЕНИЕ 13. Алгебра автоморфизмов невырожденной модельной поверхности параметризует семейство отображений одного вполне невырожденного ростка в другой.

Полученные результаты можно резюмировать в виде следующей теоремы.

TЕОрема 14. Для произвольных значений CR-размерности $n \leqslant 1$ и коразмерности $K \leqslant 1$ невырохсденная модельная поверхность $Q(n, K)$ вида (2) является хорошей модельной поверхностью невырохденного ростка гладкого вполне невырожденного вещественного подмногообразия, что выражсается в выполнении следующих свойств 1-5.

Свойство 1 (универсальность). Любой вполне невырожденньй росток многообразия типа $(n, K), n \geqslant 1, K \geqslant 1$, в $\mathbb{C}^{n+K}$ эквивалентен ростку вида $(1)$, и с каждым таким ростком можно однозначно, с точностью до эквивалентности, связать модельную поверхность $Q$ (утверждение 2 ).

Свойство 2 (конечномерность). (а) Группа голоморфных автоморфизмов невырожденной модельной поверхности является конечномерной группой Ли (утверждение 8).

(b) Критерием конечномерности групш голоморфных автоморфизмов модельной поверхности является ее невырожденность (замечание 9).

Свойство 3 (однородность). Любая модельная поверхность однородна, т.е. ее голоморфные автоморфизмы действуют на ней транзитивно, однородность обеспечивается полиномиально-треугольными преобразованиями (утверждение 3 ).

Свойство 4 (симметричность). (а) Модельная поверхность является самой симметричной невырожденной поверхностью, т.е. размерность группы ростка вполне невырожденной поверхности не превосходит размерности группы автоморфизмов ее касательной модельной поверхности (утверждение 12).

(b) Алгебра автоморфизмов модельной поверхности параметризует семейство отображений одного вполне невырожденного ростка в другой (утверждение 13).

Свойство 5 (алгебраические свойства). (а) Алгебра Ли голоморфных векторных полей на невырожденной модельной поверхности - это некоторая алгебра полиномиальных векторных полей ограниченной степени, граница зависит лишь от $n$ и $K$ (утверждение 10).

(b) Группа автоморфизмов невырожденной модельной поверхности - это групша Ли, являющаяся подгрупой группы бирациональных преобразований $\mathbb{C}^{n+K}$ ограниченной степени, оценка степени зависит лиш от $n$ и $K$ (утверждение 11$)$.

(c) Два ростка эквивалентны лиш в том случае, если эквивалентны их касательные модельные поверхности, две модельные поверхности голомор фно эквивалентны в том и только том случае, когда они эквивалентны линейно (утверждение 7).

(d) Модельная поверхность обладает естественной структурой групшы Ли (утверждение 3).

Сформулируем три вопроса.

Вопрос 1. Танака для случая $d=2$ строил доказательство конечномерности на языке градуированных алгебр. В работе Танаки [25] имеется замечание, что его подход можно реализовать для градуированных алгебр с отрицательной компонентой произвольной длины и что он осуществил аналогичное построение для типа $(1,2)$, в котором $d=3$ (эта ситуация была также рассмотрена в [7]). Было бы интересно осуществить его программу в нашей ситуации. 
Вопрос 2. В работе Наруки [25] для каждой конечномерной градуированной алгебры Ли, обладающей некоторьпи необходимьми свойствами, строится CR-многообразие (стандартное CR-многообразие), чья алгебра Леви-Танаки совпадает с исходной (см. также [27]). Было бы интересно выяснить, как соотносятся стандартные многообразия Наруки с построенньми здесь модельньми поверхностями, которые также являются многообразиями, сильно регулярньми по Танаке с конечномерной алгеброй Леви-Танаки.

Как было отмечено выше, модельные поверхности одного типа могут быть неэквивалентны и их групшы могут иметь разные размерности. Обозначим через $D_{m}(n, K)$ и $D_{m_{0}}(n, K)$ соответственно максимумы размерностей Aut $Q$ и $\operatorname{Aut}_{0} Q$, взятые по всем невырожденным модельньм поверхностям данного типа. Из доказанного следует конечность этих величин. Теперь утверждение 12 можно сформулировать так: для любого вполне невырожденного ростка $M_{\xi}$ имеют место оценки

$$
\operatorname{dim} \text { Aut } M_{\xi} \leqslant D_{m}(n, K), \quad \operatorname{dim}_{\mathrm{Aut}_{\xi}} M_{\xi} \leqslant D_{m_{0}}(n, K) .
$$

Если росток не является вполне невырожденным, то утверждение 12 непосредственно к нему не применимо. Однако имеет место следующее

ПРЕДЛОЖЕНИЕ 15. Пусть вещественно-аналитический росток $M_{\xi}$ таков, что его представитель - поверхность $M$ вполне невырохсдена в точке общего полохения, т.е. вне собственного аналитического подмнохества. Тогда $\operatorname{dim}$ Aut $M_{\xi} \leqslant D_{m}(n, K)$.

ДокАЗАтЕЛЬСтво. Пусть $X_{1}, \ldots, X_{m}$ - поля из aut $M_{\xi}$, причем $m>D(n, K)$. Перейдем от ростков к их представителям и сместимся в близкую к $\xi$ точку, в которой поверхность вполне невырождена. Из утверждения 12 получаем, что в окрестности этой точки поля линейно зависимы. Эта линейная комбинация, как следует из вещественной аналитичности, обязана быть равной нулю и в окрестности точки $\xi$. Утверждение доказано.

Вопрос 3. Для произвольного ростка в виде гипотезы (это и есть третий вопрос), можно сформулировать следующую альтернативу:

$$
\text { либо } \operatorname{dim} \text { Aut } M_{\xi} \leqslant D_{m}(n, K), \text { либо } \operatorname{dim} \text { Aut } M_{\xi}=\infty,
$$

контрпример к которой нам неизвестен.

В заключение выставим для обозрения модельные поверхности нескольких младших степеней. Итак, фиксируем некоторое $n \geqslant 1, z \in \mathbb{C}^{n}$ и положим $l_{m}=C_{2 n+m-1}^{m}-$ $2 C_{n+m-1}^{m}\left(C_{p}^{q}-\right.$ биномиальньй коэффициент).

Модельные поверхности степени $d=2$ - это хорошо известные поверхности вида

$$
\operatorname{Im} w_{2}=\langle z, \bar{z}\rangle
$$

$w_{2} \in \mathbb{C}^{K}$. Они обслуживают следующий набор коразмерностей:

$$
1 \leqslant K \leqslant n^{2} .
$$

Модельные поверхности степени $d=3$ - это поверхности вида

$$
\operatorname{Im} w_{2}=\Phi_{1, \overline{1}}(z, \bar{z})
$$




$$
\operatorname{Im} w_{3}=2 \operatorname{Re}\left\langle z^{2}, \bar{z}\right\rangle
$$

Здесь, размерность $w_{2}$ достигла максимума: $k_{2}=l_{2}=n^{2}$ и под $\Phi_{1, \overline{1}}(z, \bar{z})$ понимается произвольный базис пространства эрмитовых форм; $w_{3} \in \mathbb{C}^{K-k_{2}}, k_{3}=l_{3}=n^{2}(n+1)$. Модель обслуживает следующий набор коразмерностей:

$$
k_{2}+1 \leqslant K \leqslant k_{2}+k_{3}
$$

Модельные поверхности степени $d=4$ - это поверхности вида

$$
\begin{aligned}
& \operatorname{Im} w_{2}=\Phi_{1, \overline{1}}(z, \bar{z}), \\
& \operatorname{Im} w_{3}=2 \operatorname{Re} \Phi_{2, \overline{1}}\left(z^{2}, \bar{z}\right), \\
& \operatorname{Im} w_{4}=2 \operatorname{Re}\left(\left\langle z^{3}, \bar{z}\right\rangle+\left\langle z^{2}, \bar{z}^{2}\right\rangle\right) .
\end{aligned}
$$

Здесь

$$
\begin{aligned}
& w_{j} \in \mathbb{C}^{k_{j}}, \quad j=2,3, \quad w_{4} \in \mathbb{C}^{k}, \\
& k_{4}=l_{4}=\frac{n^{2}(n+1)(7 n+11)}{12}, \quad 1 \leqslant k \leqslant k_{4} .
\end{aligned}
$$

Набор коразмерностей:

$$
k_{2}+k_{3}+1 \leqslant K \leqslant k_{2}+k_{3}+k_{4} .
$$

Модельные поверхности степени $d=5$ - это поверхности вида

$$
\begin{aligned}
& \operatorname{Im} w_{2}=\Phi_{1, \overline{1}}(z, \bar{z}) \\
& \operatorname{Im} w_{3}=2 \operatorname{Re} \Phi_{2, \overline{1}}\left(z^{2}, \bar{z}\right) \\
& \operatorname{Im} w_{4}=2 \operatorname{Re}\left(\Phi_{3, \overline{1}}\left(z^{3}, \bar{z}\right)+\Phi_{2, \overline{2}}\left(z^{2}, \bar{z}^{2}\right)\right), \\
& \operatorname{Im} w_{5}=2 \operatorname{Re}\left(\left\langle z^{4}, \bar{z}\right\rangle+\left\langle z^{3}, \bar{z}^{2}\right\rangle+\left\langle z^{2}, \bar{z}, u_{2}\right\rangle\right) .
\end{aligned}
$$

Здесь

$$
\begin{aligned}
& w_{j} \in \mathbb{C}^{k_{j}}, \quad j=2,3,4, \quad w_{5} \in \mathbb{C}^{k}, \\
& l_{5}=\frac{n^{2}(n+1)(n+2)(3 n+5)}{12}, \quad 1 \leqslant k \leqslant k_{5}=l_{5}+l_{2} l_{3} .
\end{aligned}
$$

Набор коразмерностей:

$$
k_{2}+k_{3}+k_{4}+1 \leqslant K \leqslant k_{2}+k_{3}+k_{4}+k_{5} \text {. }
$$

Модельные поверхности степени $d=6$ - это поверхности вида

$$
\begin{aligned}
& \operatorname{Im} w_{2}=\Phi_{1, \overline{1}}(z, \bar{z}) \\
& \operatorname{Im} w_{3}=2 \operatorname{Re} \Phi_{2, \overline{1}}\left(z^{2}, \bar{z}\right) \\
& \operatorname{Im} w_{4}=2 \operatorname{Re}\left(\Phi_{3, \overline{1}}\left(z^{3}, \bar{z}\right)+\Phi_{2, \overline{2}}\left(z^{2} \bar{z}^{2}\right)\right) \\
& \operatorname{Im} w_{5}=2 \operatorname{Re}\left(\Phi_{4, \overline{1}}\left(z^{4}, \bar{z}\right)+\Phi_{3, \overline{2}}\left(z^{3}, \bar{z}^{2}\right)+\Psi_{2, \overline{1}}\left(z^{2}, \bar{z}, u_{2}\right)\right), \\
& \operatorname{Im} w_{6}=2 \operatorname{Re}\left(\left\langle z^{5}, \bar{z}\right\rangle+\left\langle z^{4}, \bar{z}^{2}\right\rangle+\left\langle z^{3}, \bar{z}^{3}\right\rangle+\left\langle z^{3}, \bar{z}, u_{2}\right\rangle+\left\langle z^{2}, \bar{z}^{2}, u_{2}\right\rangle\right) .
\end{aligned}
$$


Здесь

$$
\begin{aligned}
w_{j} & \in \mathbb{C}^{k_{j}}, \quad j=2,3,4,5, \quad w_{6} \in \mathbb{C}^{k}, \\
l_{6} & =\frac{n^{2}(n+1)(n+2)\left(31 n^{2}+132 n+137\right)}{360}, \quad 1 \leqslant k \leqslant k_{6}=l_{6}+l_{2} l_{4} .
\end{aligned}
$$

Набор коразмерностей:

$$
k_{2}+k_{3}+k_{4}+k_{5}+1 \leqslant K \leqslant k_{2}+k_{3}+k_{4}+k_{5}+k_{6} .
$$

Модельные поверхности степени $d=7$ - это поверхности вида

$$
\begin{aligned}
\operatorname{Im} w_{2}= & \Phi_{1, \overline{1}}(z, \bar{z}), \\
\operatorname{Im} w_{3}= & 2 \operatorname{Re} \Phi_{2, \overline{1}}\left(z^{2}, \bar{z}\right), \\
\operatorname{Im} w_{4}= & 2 \operatorname{Re}\left(\Phi_{3, \overline{1}}\left(z^{3}, \bar{z}\right)+\Phi_{2, \overline{2}}\left(z^{2}, \bar{z}^{2}\right)\right), \\
\operatorname{Im} w_{5}= & 2 \operatorname{Re}\left(\Phi_{4, \overline{1}}\left(z^{4}, \bar{z}\right)+\Phi_{3, \overline{2}}\left(z^{3}, \bar{z}^{2}\right)+\Psi_{2, \overline{1}}\left(z^{2}, \bar{z}, u_{2}\right)\right), \\
\operatorname{Im} w_{6}= & 2 \operatorname{Re}\left(\Phi_{5, \overline{1}}\left(z^{5}, \bar{z}\right)+\Phi_{4, \overline{2}}\left(z^{4}, \bar{z}^{2}\right)+\Phi_{3, \overline{3}}\left(z^{3}, \bar{z}^{3}\right)\right. \\
& \left.+\Psi_{3, \overline{1}}\left(z^{3}, \bar{z}, u_{2}\right)+\Psi_{2, \overline{2}}\left(z^{2}, \bar{z}^{2}, u_{2}\right)\right), \\
\operatorname{Im} w_{7}= & 2 \operatorname{Re}\left(\left\langle z^{6}, \bar{z}\right\rangle+\left\langle z^{5}, \bar{z}^{2}\right\rangle+\left\langle z^{4}, \bar{z}^{3}\right\rangle+\left\langle z^{4}, \bar{z}, u_{2}\right\rangle+\left\langle z^{3}, \bar{z}^{2}, u_{2}\right\rangle\right. \\
& \left.+\left\langle z^{2}, \bar{z}, u_{2}{ }^{2}\right\rangle+\left\langle z^{3}, \bar{z}, u_{3}\right\rangle+\left\langle z^{2}, \bar{z}^{2}, u_{3}\right\rangle\right) .
\end{aligned}
$$

Здесь

$$
\begin{aligned}
& w_{j} \in \mathbb{C}^{k_{j}}, \quad j=2,3,4,5,6, \quad w_{7} \in \mathbb{C}^{k}, \\
& l_{7}=\frac{n^{2}(n+1)(n+2)^{2}(n+3)(3 n+7)}{120}, \quad 1 \leqslant k \leqslant k_{7}=l_{7}+l_{2} l_{5}+\frac{\left(l_{2}+1\right) l_{2} l_{3}}{2}+l_{3} l_{4} .
\end{aligned}
$$

Набор коразмерностей:

$$
k_{2}+k_{3}+k_{4}+k_{5}+k_{6}+1 \leqslant K \leqslant k_{2}+k_{3}+k_{4}+k_{5}+k_{6}+k_{7} .
$$

Напомним, что повсюду в правой части, за исключением последнего соотношения, стоят базисные наборы многочленов из соответствующих пространств, а в последнем соотношении стоит произвольный линейно независимый набор.

\section{СПИСОК ЦИТИРОВАННОЙ ЛИТЕРАТУРЫ}

[1] Burns D., Shnider S., Wells R. O. Deformation of strictly pseudoconvex domains // Invent. Math. 1978. V. 46. №3. P. 199-217.

[2] Cartan E. Sur la geometrie pseudoconforme des hypersurfaces de deux variables complexes // Ann. Math. Pura Appl. 1932. V. 11. №4. P. 17-90 (Oeuvres II, V. 2, P. 1231-1304).

[3] Chern S. S., Moser J. K. Real hypersurfaces in complex manifold // Acta Math. 1974. V. 133. № 3-4. P. 219-271.

[4] Белошапка В. К. Вещественные подмногообразия комплексного пространства их полиномиальные модели, автоморфизмы и проблемы классификации // УМН. 2002. Т. 57. №1. C. $3-44$. 
[5] Tanaka N. On the pseudo-conformal geometry of hypersurfaces of the space of $n$ complex variables // J. Math. Soc. Japan. 1962. V. 14. P. 397-429.

[6] Белошапка В. К. О голоморфных преобразованиях квадрики // Матем. сб. 1991. Т. 182. № 2. C. 203-219.

[7] Beloshapka V.K. CR-varieties of the type $(1,2)$ as varieties of "super-high" codimension // Russian J. Math. Phys. 1998. V. 5. № 2. P. 399-404.

[8] Шананина Е. Н. Модели CR-многообразий типа $(1, k)$ при $3 \leqslant k \leqslant 7$ и их автоморфизмы // Матем. заметки. 2000. Т. 67. №3. С. 452-459.

[9] Белошапка В.К. Кубическая модель вещественного многообразия // Матем. заметки. 2001. T. 70. № 4. C. 503-519.

[10] Белошапка В. К. Полиномиальные модели вещественных многообразий // Изв. РАН. Сер. матем. 2001. Т. 65. № 4. С. 3-20.

[11] Белошапка В. К. Квазипериодическая система полиномиальных моделей CR-многообразий // Тр. МИАН. 2001. Т. 235. С. 7-35.

[12] Шананина Е. Н. Полиномиальные модели степени 5 и алгебры их автоморфизмов // Матем. заметки (в печати). 2003.

[13] Kohn J. J. Boundary behavior of $\bar{\partial}$ on weakly pseudoconvex manifolds of dimension two // J. Differential Geom. 1972. V. 6. P. 553-542.

[14] Bloom T., Graham I. On type conditions for generic real submanifolds in $\mathbb{C}^{n} / /$ Invent. Math. 1977. V. 40. P. 217-243.

[15] Белошапка В.К. Функции, плюригармонические на многообразии // Изв. АН СССР. Сер. матем. 1978. Т. 12. №3. С. 439-447.

[16] Туманов А. Е. Продолжение CR-функций с многообразий конечного типа в клин // Матем. сб. 1988 . Т. 136 . С. $129-140$.

[17] Baouendi M.S., Huang X., Rothschild L. P. Regularity of CR-mappings between algebraic hypersurfaces // Invent. Math. 1996. V. 125. P. 13-36.

[18] Baouendi M.S., Ebenfelt P., Rothschild L. P. Real Submanifolds in Complex Space and Their Mappings. Princeton Math. Ser. V. 47. Princeton, NJ: Princeton Univ. Press, 1999.

[19] Белошапка В.K. Геометрические инварианты CR-многообразий // Матем. заметки. 1994. T. 55. № 5. C. 3-12.

[20] Garrity T., Mizner R. Invariants of vector-valued and sesquilinear forms // Linear Algebra Appl. 1995. V. 218. P. 225-237.

[21] Белошапка В. К. Инварианты CR-многообразий, связанные с касательной квадрикой // Матем. заметки. 1996. Т. 59. №1. С. 42-52.

[22] Белошапка В. К. Локальные инварианты и запреты на отображения CR-многообразий // Матем. заметки. 1996. Т. 60. № 4. С. 588-592.

[23] Zaitsev D. Germs of local automorphisms of real-analytic CR structures and analytic dependence on k-jets // Math. Res. Lett. 1997. V. 4. P. 1-20.

[24] Туманов A. Е. Конечномерность группы CR-автоморфизмов стандартного CR-многообразия и собственные голоморфные отображения областей Зигеля // Изв. АН СССР. 1988. Т. 52. № 3. C. 651-659.

[25] Tanaka N. On generalized graded Lie algebras and geometric structures I // J. Math. Soc. Japan. 1967. V. 19. № 2. P. 215-254.

[26] Naruki I. Holomorphic extension problem for standart real submanifolds of second kind // Publ. Res. Inst. Math. Kyoto Univ. 1970. V. 6. №1. P. 113-187.

[27] Medori C., Nacinovich M. Maximally homogeneous nondegenerate CR-manifolds // Adv. Geom. 2001. V. 1. P. 89-95.

[28] Tanaka N. Graded Lie algebras and geometric structures // Proc. US-Japan Seminar in Differential Geometry (Kyoto, 1965). Tokyo: Nippon Hyoronsha, 1965. P. 147-150.

Московский государственный университет им. М. В. Ломоносова

E-mail: vkb@strogino.ru 